\title{
Electromyogram (EMG) based Fingers Movement Recognition Using Neighborhood Preserving Analysis with QR-Decomposition
}

\author{
Rami N. Khushaba ${ }^{\# 1}$, Sarath Kodagoda ${ }^{* 2}$, Dikai Liu ${ }^{\# 3}$, Gamini Dissanayake ${ }^{\# 4}$ \\ \# Faculty of Engineering and Information Technology, University of Technology, Sydney (UTS) \\ 15 Broadway, Ultimo, NSW, Australia \\ ${ }^{1}$ Rami.Khushaba@uts.edu.au \\ ${ }^{2}$ Sarath.Kodagoda@uts.edu.au \\ ${ }^{3}$ Dikai.Liuduts.edu.au \\ ${ }^{4}$ Gamini.Dissanayake@uts. edu. au
}

\begin{abstract}
Surface Electromyogram (EMG) signals recorded from an amputee's residual muscles have been investigated as a source of control for prosthetic devices for many years. Despite the extensive research focus on the EMG control of arm and gross hand movements, more dexterous individual and combined prosthetic fingers control has not received the same amount of attention. To facilitate such a control scheme, the first and the most significant step is the extraction of a set of highly discriminative feature set that can well separate between the different fingers movements and to do so in a computationally efficient manner. In this paper, an accurate and efficient feature projection method based on Fuzzy Neighborhood Preserving Analysis (FNPA) with QR-decomposition, is proposed and denoted as FNPA. Unlike existing attempts in fuzzy linear discriminant analysis, the objective of the proposed FNPA is to minimize the distance between samples that belong to the same class and maximize the distance between the centers of different classes, while taking into account the contribution of the samples to the different classes. The method also aims to efficiently overcome the singularity problems of classical LDA and Fuzzy LDA. The proposed FNPA is validated on EMG datasets collected from nine subjects performing 10 classes of individual and combined fingers movements. Practical results indicate the significance of FNPA in comparison to many other feature projection methods with an average accuracy of $91 \%$, using only two EMG electrodes.
\end{abstract}

\section{INTRODUCTION}

Powered upper limb prostheses can be controlled using surface Electromyogram (EMG) signals detected from the residual muscles in a scheme denoted as myoelectric control. In such a control scheme, voluntarily controllable features of the surface EMG are used as input to select and modulate the functions of a powered prosthesis. This approach has clinical applications in individuals with amputations or congenitally deficient upper limbs [1]. Typically a pattern recognition framework is utilized to classify the acquired EMG signals into one of a predefined sets of forearm movements [2], [3]. Various feature sets and classification methods have been utilized in the literature demonstrating the feasibility of myoelectric control [4]. Given the success of utilizing EMG signals in decoding the intended forearm movements, there have been recent attempts to achieve more dexterous individual finger control [5], [6]. For example, Peleg et al. [7] employed surface EMG signals to identify when and which finger is activated using only two electrodes placed on the forearm. Tsenov et al. [8] also utilized two EMG electrodes to detect four finger movements using time domain features and neural networks classifiers achieving nearly $93 \%$ accuracy. However, both of these attempts did not consider combined fingers movements. Tenore et al. [5] extended the idea of EMG based finger control into movements that consisted of flexion and extension of all the fingers individually and of the middle, ring and little finger as a group achieving $\geq 98 \%$ accuracy with thirty-two electrodes [5], [9] and with fifteen electrodes [10]. However, a reduction in the number of electrodes, without compromising the classification accuracy, would significantly simplify the requirements for controlling state of the art prostheses.

While much of the work presented in literature focus on experiments with able-bodied subjects, Cipriani et al. [11] reports real-time experiments on both able-bodied and amputees participants. Eight pairs of electrodes were utilized to classify seven finger movements, including two classes of combined fingers movements. A $k$-nearest neighbor $(k \mathrm{NN})$ classifier achieved an average classification accuracy of $79 \%$ (for amputees)-to-89\% (for able-bodied participants). However, no experiments were conducted to validate the need for the total eight pairs of electrodes upon that of a smaller combination. Additionally, the $k \mathrm{NN}$ classifier requires large memory to store all the training patterns to compare each testing sample based on distances, while further computing difficulties are imposed on $k \mathrm{NN}$ by the high dimensional data.

Due to the high variance nature of the EMG signal [12], the extracted information from the EMG signals tend to be liberally dispersed amongst the original feature set. In such a case, feature projection based dimensionality reduction methods were shown to be able to consolidate this information in an effective manner by a number of researchers [12], [13], [14], [15]. The use of the projection methods is also justified by the multichannel approach that is usually utilized to capture motor information from different muscles leading to a large feature set that even affects the total time of classification. 
To this end, various projection methods were utilized in EMG classification including: Principal Components Analysis (PCA) [13], Linear Discriminant Analysis (LDA) [12], Uncorrelated Linear Discriminant Analysis (ULDA) [16], [14], and very recently Orthogonal Fuzzy Neighborhood Discriminant Analysis (OFNDA) [15]. However, each one of these methods has its own limitations, as will be described in the next section.

In this paper, a new method denoted as FNPA is proposed based on earlier work of the first author in Khushaba et al. [15]. The key difference between OFNDA and FNPA lies in the way by which the well-known singularity of classical LDA (and its variants) is avoided. Specifically, FNPA applies the QR decomposition to modify the optimization process to avoid singularity, while OFNDA applies PCA as a preprocessing step to remove any correlation that causes singularity. Additionally, FNPA removes the orthogonal constraint on the resultant features and utilizes the QR-decomposition to solve singularity rather than to produce a set of orthogonal features.

The structure of this paper is as follows: Section II presents the proposed method and its associated derivations. Section III describes the data collection procedure and the utilized hardware. Section IV presents the experimental results and finally, conclusions are provided in Section V.

\section{FuZZY NEIGHBORHOOd DisCRIMINANT ANALYSIS WITH QR-DECOMPOSITION}

In this section, a new dimensionality reduction method is described that is considered as a novel variation to fuzzy LDA (FLDA). FLDA is a well known data-analytic tool for studying the class relationship between data points, but a major disadvantage of FLDA is that it fails to discover the local geometrical structure of the data points. It has been shown in the literature that in many cases the local structure is generally more important than global structure for discriminant analysis [17], [18], [19]. Additionally, preserving the local structure allows the maximization of the margin between data points from different classes at each local area. Specifically, a new feature projection method is proposed in which the data points are mapped into a subspace in which the nearby points with the same label are close to each other while the nearby points with different labels are far apart.

Given a universal set with elements $\mathbf{x}_{k}$ distributed in a pattern space as $\mathrm{X}=\left\{\mathbf{x}_{1}, \mathbf{x}_{2}, \ldots, \mathbf{x}_{l}\right\}$, where $k=1,2, \ldots, l$ with $l$ being the total number of patterns. For simplicity, It will be useful to describe the membership value that the $k$ 'th vector has in the $i$ 'th class with the following notation

$$
\mu_{i k}=\mu_{i}\left(\mathbf{x}_{k}\right) \in[0,1]
$$

Denote the mean of the data samples that belong to class $i$ as $\overline{\mathbf{x}_{i}}$ and the radius of the data as $r$

$$
r=\max \left\|\overline{\mathbf{x}}_{i}-\mathbf{x}_{k}\right\|_{\sigma}
$$

Then the fuzzy membership $\mu_{i k}$ can be calculated as follows

$$
\mu_{i k}=\left(\frac{\left\|\overline{\mathbf{x}}_{i}-\mathbf{x}_{k}\right\|_{\sigma}}{r+\epsilon}\right)^{\frac{-2}{m-1}}
$$

where $m$ is the fuzzification parameter, $\epsilon>0$ is a small value to avoid division by zero, and $\sigma$ is the standard deviation involved in the distance computation. Finally, the membership of each of the samples in all of the problem classes is normalized according to $\sum_{i=1}^{c} \mu_{i k}=1$.

The description of the proposed FNPA proceeds with the fuzzy within class scatter matrix given as:

$$
S_{W}=\sum_{i=1}^{c} \sum_{k=1}^{l_{i}} \mu_{i k}\left(\mathbf{x}_{k}-\mathbf{v}_{i}\right)\left(\mathbf{x}_{k}-\mathbf{v}_{i}\right)^{T}
$$

where $\mu_{i k}$ is the membership of pattern $k$ in class $i, \mathbf{x}_{k}$ is the $k$ 'th sample, $\mathbf{v}_{i}$ is the mean of the patterns that belong to class $i$.

$$
\mathbf{v}_{i}=\frac{\sum_{k=1}^{l_{i}} \mu_{i k} \mathbf{x}_{k}}{\sum_{k=1}^{l_{i}} \mu_{i k}}
$$

We proceed with the modification of the within class scatter matrix starting from Eq.4 as follows

$$
\begin{array}{r}
S_{W}=\sum_{i=1}^{c} \sum_{k=1}^{l_{i}} \mu_{i k}\left(\mathbf{x}_{k} \mathbf{x}_{k}^{T}-\mathbf{v}_{i} \mathbf{x}_{k}^{T}-\mathbf{x}_{k} \mathbf{v}_{i}^{T}+\mathbf{v}_{i} \mathbf{v}_{i}^{T}\right) \\
S_{W}=\sum_{i=1}^{c}\left[\sum_{k=1}^{l_{i}} \mu_{i k} \mathbf{x}_{k} \mathbf{x}_{k}^{T}-\mathbf{v}_{i} \sum_{k=1}^{l_{i}} \mu_{i k} \mathbf{x}_{k}^{T}\right. \\
\left.-\mathbf{v}_{i}^{T} \sum_{k=1}^{l_{i}} \mu_{i k} \mathbf{x}_{k}+\mathbf{v}_{i} \mathbf{v}_{i}^{T} \sum_{k=1}^{l_{i}} \mu_{i k}\right]
\end{array}
$$

Using Eq.5 we replace $\sum_{k=1}^{l_{i}} \mu_{i k} \mathbf{x}_{k}^{T}$ with $\mathbf{v}_{i}^{T} \sum_{k=1}^{l_{i}} \mu_{i k}$, and $\sum_{k=1}^{l_{i}} \mu_{i k} \mathbf{x}_{k}$ with $\mathbf{v}_{i} \sum_{k=1}^{l_{i}} \mu_{i k}$. Thus Eq.7 can be re-written as

$$
S_{W}=\sum_{i=1}^{c} \frac{1}{2 \sum_{j=1}^{l_{i}} \mu_{i j}} \sum_{k=1}^{l_{i}} \sum_{j=1}^{l_{i}} \mu_{i k} \mu_{i j}\left(\mathbf{x}_{k}-\mathbf{x}_{j}\right)\left(\mathbf{x}_{k}-\mathbf{x}_{j}\right)^{T}
$$

The above equation indicates that the objective of the new $S_{W}$ is to minimize the distance between samples of the same class when projecting them. Such distance can be limited to that between each sample and its $k$-nearest neighbors, thus preserving the local neighborhood information. Additionally, in a similar manner to FLDA, the proposed new $S_{W}$ also incorporates the membership values thus considering the samples contribution in the class when preserving their distances. According the strong law of large numbers [20], Eq. 8 can be further simplified into one that employs matrices multiplications rather than using summations. In order to simplify the above equation and the subsequent equations, we provide the following two definitions 
Definition-1: $B_{i}$ is the fuzzy amount of elements in fuzzy class $c_{i}$, this is equal to the summation of the membership values of the samples that belong to class $c_{i}$ in the same class, and is given by:

$$
B_{i}=\sum_{k=1}^{l_{i}} \mu_{i k}
$$

Definition-2: $N$ is the fuzzy amount of elements in all fuzzy classes, and this is given by:

$$
N=\sum_{i=1}^{c} B_{i}
$$

If we replace $\mu_{i k} \mu_{i j}$ in Eq. 8 by $W_{k j}$ and given that we already have the exterior sum in terms of $i$, then

$$
\begin{aligned}
& S_{W}=\sum_{i=1}^{c} \frac{1}{2 B_{i}} \sum_{k=1}^{l_{i}} \sum_{j=1}^{l_{i}}\left(\mathbf{x}_{k}-\mathbf{x}_{j}\right)\left(\mathbf{x}_{k}-\mathbf{x}_{j}\right)^{T} W_{k j} \\
& S_{W}=\sum_{i=1}^{c} \frac{2}{2 B_{i}}\left(\sum_{k}^{l_{i}} \mathbf{x}_{k} \mathbf{x}_{k}^{T} D_{k k}-\sum_{k, j}^{l_{i}} \mathbf{x}_{k} \mathbf{x}_{j}^{T} W_{k j}\right)
\end{aligned}
$$

which can be generalized to

$$
\begin{gathered}
S_{W}=\left(X D X^{T}-X W X^{T}\right) \\
S_{W}=\left(X L_{1} X^{T}\right)
\end{gathered}
$$

where $L_{1}=D-W$ is derived in the same manner as the Laplacian matrix in [20]. $D$ is a diagonal matrix; its entries are column sums of $W$ (or row sums since $W$ is symmetric), $D_{k k}=\sum_{k} W_{k j}$.

Now in a similar approach to the derivation of $S_{W}$, we derive the between class scatter matrix starting with the fuzzy version of $S_{B}$,

$$
S_{B}=\sum_{i=1}^{c} \sum_{k=1}^{l_{i}} \mu_{i k}\left(\mathbf{v}_{i}-\overline{\mathbf{x}}\right)\left(\mathbf{v}_{i}-\overline{\mathbf{x}}\right)^{T}
$$

where $\overline{\mathbf{x}}$ is the mean of the training samples, this is in turn given as

$$
\overline{\mathbf{x}}=\frac{\sum_{i=1}^{c} \sum_{k=1}^{l_{i}} \mu_{i k} \mathbf{x}_{k}}{\sum_{i=1}^{c} \sum_{k=1}^{l_{i}} \mu_{i k}}
$$

The between class scatter matrix represented by Eq.15 can be expressed as

$$
S_{B}=\frac{1}{2 N} \sum_{i=1}^{c} \sum_{j=1}^{c} B_{i} B_{j}\left(\mathbf{v}_{i}-\mathbf{v}_{j}\right)\left(\mathbf{v}_{i}-\mathbf{v}_{j}\right)^{T}
$$

The above equation can be also written in terms of matrices multiplications using the same approach employed with $S_{W}$. If we simply replace $B_{i} B_{j}$ with $B_{i j}$ then

$$
\begin{gathered}
S_{B}=\frac{1}{2 N} \sum_{i=1}^{c} \sum_{j=1}^{c} B_{i j}\left(\mathbf{v}_{i}-\mathbf{v}_{j}\right)\left(\mathbf{v}_{i}-\mathbf{v}_{j}\right)^{T} \\
S_{B}=\left(\sum_{i, j=1}^{c} B_{i j} \mathbf{v}_{i} \mathbf{v}_{i}^{T}-2 \sum_{i, j=1}^{c} \mathbf{v}_{i} \mathbf{v}_{j} B_{i j}+\sum_{i, j=1}^{c} \mathbf{v}_{j} \mathbf{v}_{j}^{T} B_{i j}\right)^{T} \\
S_{B}=\left(V E V^{T}-V B V^{T}\right)
\end{gathered}
$$

which can be written as

$$
S_{B}=V L_{2} V^{T}
$$

where $L_{2}=E-B$ and $E$ is a diagonal matrix; its entries are column (or row since $B$ is symmetric) sums of $B, E_{i i}=$ $\sum_{i} B_{i j}$. The $i$ 'th column of matrix $\mathbf{V}$ is $\mathbf{V}_{i}$. Using the new representation of $S_{W}$ and $S_{B}$ given by Eq.14 and Eq.21, the transformation matrix $G_{F N P A}$ related to FNPA is found as the eigen vectors of Eq.22

$$
\mathbf{G}_{F N P A}=\arg \max _{G} \operatorname{trace}\left(\frac{\mathbf{G}^{T} S_{B} \mathbf{G}}{\mathbf{G}^{T} S_{W} \mathbf{G}}\right),
$$

where the above function aims at maximizing the distance between the samples of different classes while minimizing the distance between the samples of the same class.

\section{A. QR-Decomposition based FNPA}

In most real world problems we usually end up with singular matrices and thus the above optimization in Eq.22 may simply fails. To overcome the singularity problem, a PCA preprocessing step was utilized in [15]. However, its generally known that PCA does not consider the class information when projecting the data. Thus, important information may be lost during the PCA transformation step. Unlike previous work, we propose a modified optimization function upon that of Eq.22 in the following manner:

A closer look at Eq.13 and Eq.20 reveals that Eq.22 can be maximized by minimizing $B$ and maximizing $W$. Thus, to simplify the optimization in Eq.22, the following steps are adopted:

- Normalize the matrix $W$ such that each column summation becomes 1 (or row since its symmetric). This in turn makes the diagonal matrix $D$ an identity one and $L_{1}$ become equivalent to $I-W$, with $I$ being an identity matrix.

- Take the second part of the denominator represented by $X W X^{T}$ to the numerator and change the sign. This is justified by that minimizing this term at the denominator is equivalent to maximizing it at the numerator. Thus, the denominator simplifies to $X X^{T}$ while the numerator is given as $\alpha V L_{2} V^{T}+(1-\alpha) X W X^{T}$, where $\alpha$ with $1>\alpha>0$ is a parameter introduced here to regulate the 
importance of the information represented by $X W X^{T}$ with respect to the information represented by the different classes centers defined in $V$.

- Then we rewrite the first part of the new numerator value from $V L_{2} V^{T}$, with $V$ being the matrix holding the different classes mean, into $\alpha X U^{T} L_{2} U X^{T}+(1-\alpha) X W X^{T}$, with $U$ being the matrix that holds the membership values of each of the samples in all of the problem classes according to the definition given by Eq.3 and Eq.5.

Thus, the above objective function in Eq.22 simplifies to

$$
\arg \max _{G} \operatorname{trace}\left(\frac{G^{T} X A X^{T} G}{G^{T} X X^{T} G}\right)
$$

with $A=\alpha U^{T} L_{2} U+(1-\alpha) W$. In order to avoid singularity, we propose here to employ the QR-decomposition as one possible solution. Let $X=Q R$ be the QR-decomposition of $X$, where $Q \in \mathbb{R}^{n \times r}$ is orthogonal matrix, with $Q^{T} Q=$ $Q Q^{T}=I$, and $R \in \mathbb{R}^{r \times l}$ is upper triangular (with $r=$ $\operatorname{rank}(X))$. Thus, Eq.22.

$$
\arg \max _{G} \operatorname{trace}\left(\frac{G^{T} Q R A R^{T} Q^{T} G}{G^{T} Q R R^{T} Q^{T} G}\right)
$$

Assuming an optimal transformation matrix $H=Q^{T} G$, for some $G \in \mathbb{R}^{r \times q}$, i.e., extracting the $q$-leading features, then Eq. 24 can be written as

$$
\arg \max _{H} \operatorname{trace}\left(\frac{H^{T} R A R^{T} H}{H^{T} R R^{T} H}\right)
$$

The optimal solution in this case (i.e., the columns of $H^{*}$ ) are the $q$-leading eigenvectors of the above equation associated with the $q$ largest eigenvalues. After getting the $H^{*}$ then we simply multiply $H^{*}$ by $Q$ to get the final projection matrix, i.e., $G^{*}=H^{*} Q$.

\section{DATA COLlection}

Nine subjects, seven males and two females, aged between 20-35 years were recruited to perform the required fingers movements. The subjects were all normally limbed with no neurological or muscular disorders. All participants provided informed consent prior to participating in the study. Subjects were seated on an armchair, with their arm supported and fixed at one position to avoid the effect of different limb positions on the generated EMG signals.

The EMG data was collected using two EMG channels (Delsys DE 2.x series EMG sensors) and processed by the Bagnoli Desktop EMG Systems from Delsys Inc. A 2-slot adhesive skin interface was applied on each of the sensors to firmly stick the sensors to the skin. A conductive adhesive reference electrode (Dermatrode Reference Electrode) was utilized on the wrist of each subject. The positions of these electrodes are shown in Fig.1. The EMG signals collected from the electrodes were amplified using a Delsys Bagnoli- 8 amplifier to a total gain of 1000. A 12-bit analog-to-digital converter (National Instruments, BNC-2090) was used to sample the signal at $4000 \mathrm{~Hz}$; the signals were acquired using Delsys EMGWorks
Acquisition software. The EMG signals were then bandpass filtered between $20-450 \mathrm{~Hz}$ with a notch filter implemented to remove the $50 \mathrm{~Hz}$ line interference.

Ten classes of individual and combined fingers movements were implemented including: the flexion of each of the individual fingers, i.e., Thumb (T), Index (I), Middle (M), Ring (R), Little (L) and the pinching of combined Thumb-Index (T-I), Thumb-Middle (T-M), Thumb-Ring (T-R), Thumb-Little (T-L), and finally the hand close (HC) as shown in Fig.2.

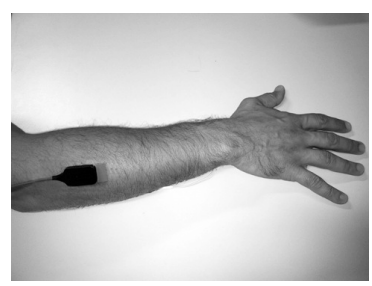

(a) position of first electrode

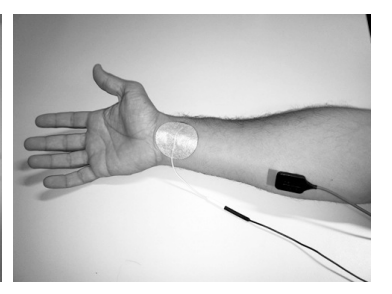

(b) position of second electrode
Fig. 1. Electrodes placement on the right forearm

When collecting data, the subjects were asked to perform each of the aforementioned ten movements, and hold that movement for a period of 5 seconds in each trial. Six trials, or repetitions, of each movement were collected. Four trials from each movement data were allocated for training and the remaining 2 trials were allocated for testing.

\section{EXPERIMENTS AND RESULTS}

In the EMG-pattern recognition system, an analysis window size of $128 \mathrm{msec}$ that was incremented by $25 \mathrm{msec}$ was utilized when extracting the features. Various features in time and frequency domain were extracted from each of the analysis windows to represent the EMG activity. These included all of the followings (reader can refer to [4] for details): number of zero crossings (1 feature), waveform length (1 feature), number of slop-sign changes ( 1 feature), skewness (1 feature), root-mean-square ( 1 feature), mean absolute value ( 1 feature), integral absolute value (1 feature), parameters of an autoregressive (AR) model with an order of 11 providing significant enhancements upon smaller model orders (11 features), and the Hjorth time-domain parameters (3 features) totaling 21 features/channel. In a problem of 2 channels, the total number of extracted features is 42 features $(21$ feature/channel $\times 2$ channels $=42$ features).

In the feature projection step, the performance of the proposed FNPA method is validated against a set of different other feature projection methods including: PCA, Neighborhood Preserving Embedding (NPE) [17], Locality Preserving Projection (LPP) [18], Local Fisher Discriminant Analysis (LFDA)[23], Fuzzy Linear Discriminant Analysis (FLDA) [24], Uncorrelated Discriminant Analysis (ULDA) [16], and the Baseline without feature projection. All of the aforementioned methods were limited to produce only $c-1$ features (the default in discriminant analysis) to produce a competent and fair comparison of the power of these methods and their discriminatory power embedded in the first $c-1$ features. 


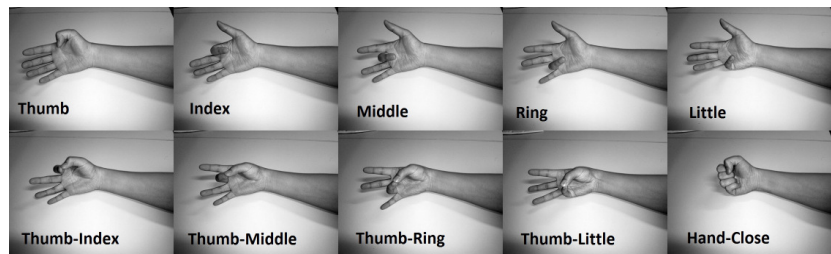

Fig. 2. Different movement classes considered in this paper.

In the classification step, different classifiers were utilized in the experiments including: Support Vector machine (SVM) with the LIBSVM implementation [21], Regression Tree classifier (RegTree) available in Matlab, and the Extreme Leaning Machine (ELM) classifier [22]. The LIBSVM classifier's parameters were optimized as: (SVM type: C-SVC), cost parameter for C-SVC was $c=8$ and kernel type was set to a radial basis function with $\gamma=12 /$ Number of features. On the other hand, the number of hidden nodes in ELM was set to 50. In the final step, a majority vote postprocessing step was utilized to smooth the output of the classifier and to further enhance the classification accuracy [14]. For a given decision point $d_{i}$, the majority vote decision smooths the classifier output by also considering the previous $m$ decisions $(m=8$ in this experiment). The value of $d_{m v}$ is simply the class label with the greatest number of occurrences in this point window of the decision stream. The classification error rates using the aforementioned feature projection methods with the above classifiers are shown in Fig.3.

The classification error rates show few important points including: Firstly, there is an obvious enhancement in classification results when using all of the LPP, LFDA, FLDA, ULDA, and FNPA feature projection methods in comparison to that achieved by the Baseline (without projection). Secondly, the performance of both PCA and NPE was fluctuating and showing higher classification error rates, on average, than the other methods and the Baseline. This is justified by that both PCA and NPE required more features to be extracted than that of $c-1$ to produce similar results to the other selected projection methods. Thirdly, it is also very clear that the only projection method that produced competent and very close results to the proposed FNPA was ULDA. A two-way analysis of variance (ANOVA) test was utilized with a significance level of $p=0.05$ as shown in Table.I, with both FNPA and ULDA showing significant enhancement on other methods.

Despite the similar results achieved by ULDA and FNPA, it should be noted here that ULDA is very expensive in terms of its associated computational cost due to the use of the singular value decomposition in its implementation (available at http: //www-users.cs.umn.edu/ jieping/uLDA/ULDA.m). Thus, to prove the significant computational time difference between ULDA and the proposed FNPA, the computational time of all of the utilized projection methods was calculated within Matlab on a PC with $3 \mathrm{GHz} \mathrm{CPU}$ and $8 \mathrm{~GB}$ of RAM as shown in Table.II. According to the computational time of different methods and their achieved classification error rates that favored FNPA and ULDA upon other projection methods, then one can clearly notice that FNPA is actually preferred upon ULDA as it provides a saving in computational cost of $(5.2865-0.9671) / 5.2865 * 100=81.70 \%$.

In the last part of the experiments, we also look at the recognition rates of the individual classes of fingers movements. In order to achieve that, the confusion matrix showing the class-wise classification accuracies was computed using the LIBSVM classifier (as it showed the best results) and averaged across all nine subjects as shown in Fig.4. According to these results, it can be noted here that there were some difficulties in separating the individual fingers movements from that of the same finger movement when combined with the thumb. Such misclassification result may be justified by the difficulty in separating the patterns associated with movements that incur large degrees of nonlinear overlapping among each other. Thus, nonlinear feature extraction methods should be investigated in a future work in this area.

\section{CONCLUSION}

An efficient feature projection method based on Fuzzy and neighborhood discriminant analysis was proposed in this paper. The proposed FNPA employed the QR-decomposition to solve the singularity problem. A two channel EMG pattern recognition system was then implemented with datasets collected from nine subjects performing 10 classes of individual and combined fingers movement. The classification results showed that both of the proposed FNPA and the well-known ULDA achieved very similar results on the collected datasets with $91 \%$ accuracy on average across all subjects. However, FNPA also showed much lower computational time requirements than that of ULDA which further proves the significance of the proposed method.

\section{REFERENCES}

[1] P.A. Parker, K. B. Englehart, and B. Hudgins, "Myoelectric signal processing for control of powered limb prostheses", Journal of Electromyography and Kinesiology, vol. 16, no. 6, pp. 541-548, 2006.

[2] K. Englehart and B. Hudgins, "A robust, real time control scheme for multifunction myoelectric control", IEEE Transactions on Biomedical Engineering, vol. 50, no. 7, pp. 848-854, 2003.

[3] M. A. Oskoei and H. Hu, "Support vector machine-based classification scheme for myoelectric control applied to upper limb", IEEE Transactions on Biomedical Engineering, vol. 55, no. 8, pp. 1956-1965, 2008.

[4] M. A. Oskoei and H. Hu, "Myoelectric control systems-A survey", Biomedical Signal Processing and Control, vol. 2, no. 4, pp. 275-294, 2007.

[5] F. Tenore, A. Ramos, A. Fahmy, S. Acharya, R. Etienne-Cummings, and N. V. Thakor, "Toward the control of individual fingers of a prosthetic hand using surface EMG signals", Proceedings of the 29th Annual International Conference of the IEEE EMBS, pp.6146-6149, 2007.

[6] R. J. Smith, D. Huberdeau, F. Tenore, and N. V. Thakor, "Real-time myoelectric decoding of individual finger movements for a virtual target task", Proceedings of the 31st Annual International Conference of the IEEE EMBS, pp. 2376-2379, 2009.

[7] D. Peleg, E. Braiman, E. Yom-Tov, and G. F. Inbar, "Classification of finger activation for use in a robotic prostheses arm", IEEE Transactions on Biomedical Engineering, vol. 10, no. 4, pp. 290-293, 2002.

[8] G. Tsenov, A. H. Zeghbib, F. Palis, N. Shoylev, V. Mladenov, "Neural networks for online classification of hand and finger movements using surface EMG signals", Proceedings of the 8th Seminar on Neural Network Applications in Electrical Engineering, pp. 167-171, 2006. 


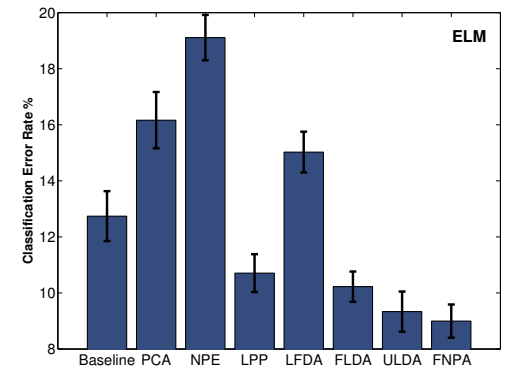

(a) ELM classifier

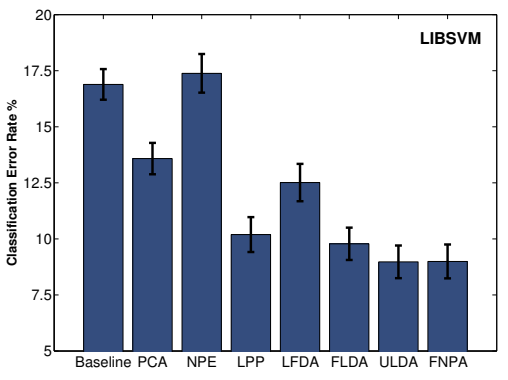

(b) LIBSVM classifier

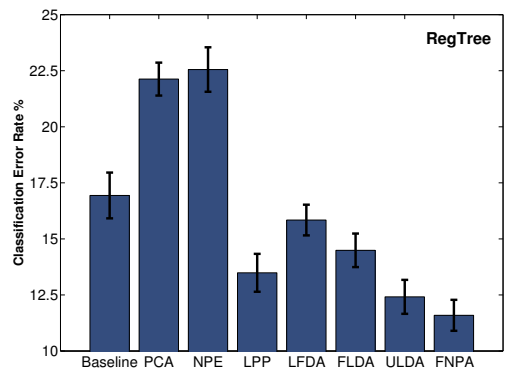

(c) RegTree classifier

Fig. 3. Average classification error rates achieved across nine subjects using different feature projection methods with the ELM, LIBSVM and the RegTree classifiers

TABLE I

TWO-WAY ANALYSIS OF VARIANCE TEST RESULTS

\begin{tabular}{|l|c|c|c|c|c|c|c|}
\hline FNPA vs. $\rightarrow$ & Baseline & PCA & NPE & LPP & LFDA & FLDA & ULDA \\
\hline ELM & 0.0096 & 0.0048 & $<0.001$ & 0.0147 & $<0.001$ & 0.0157 & 0.3046 \\
\hline LIBSVM & 0.0005 & 0.0033 & $<0.001$ & 0.0261 & $<0.001$ & 0.0327 & 0.8850 \\
\hline RegTree & 0.0019 & $<0.001$ & $<0.001$ & 0.0437 & $<0.001$ & $<0.001$ & 0.2722 \\
\hline
\end{tabular}

TABLE II

COMPUTATIONAL TIME REQUIRED B Y DIFFERENT METHODS IN SECONDS WITHIN MATLAB

\begin{tabular}{|c|c|c|c|c|c|c|}
\hline PCA & NPE & LPP & LFDA & FLDA & ULDA & FNPA \\
\hline 0.0055 & 2.0376 & 0.3816 & 0.9393 & 1.1685 & 5.2865 & 0.9671 \\
\hline
\end{tabular}

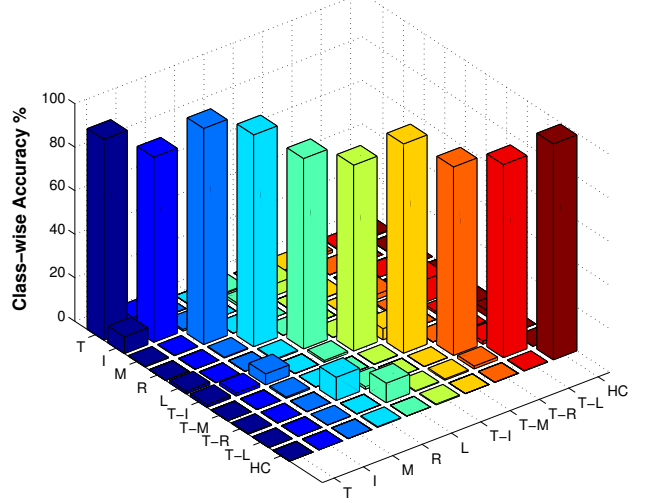

Fig. 4. Average confusion matrix showing classwise accuracy.

[9] F. V. G. Tenore, A. Ramos, A. Fahmy, S. Acharya, R. Etienne-Cummings, N. V. Thakor, "Deconding of individual finger movements using surface electrmyography", IEEE Transactions on Biomedical Engineering, vol. 56, no. 5, pp. 1427-1434, 2009.

[10] R. J. Smith, F. Tenore, D. Huberdeau, R. Etienne-Cummings, and N. V. Thakor, "Continuous decoding of finger position from surface EMG signals for the control of powered prostheses", Proceedings of the 30th Annual International Conference of the IEEE EMBS, pp. 197-200, 2008.

[11] C. Cipriani, C. Antfolk, M. Controzzi, G. Lundborg, B. Rosen, M. C. Carrozza, and F. Sebelius, "Online myoelectric control of a dexterous hand prosthesis by transradial amputees", IEEE Transactions on Neural Systems and Rehabilitation Engineering, vol. PP, no. 99, DOI:10.1109/TNSRE.2011.2108667, 2011.

[12] J. U. Chu, I. Moon, M. S. Mun, "A supervised feature projection for realtime multifunction myoelectric hand control", In Proc. 28th IEEE EMBS Annual International Conference, New York, USA, 2006, pp. 2417-2420.
[13] K. Englehart, "Signal representation for classification of the transient myoelectric signal", PhD dissertation, Department of Electrical and Computer Engineering, University of New Brunswick, New Brunswick, Canada, 1998.

[14] A. D. C. Chan and G. C. Green, "Myoelectric control development toolbox", In Proc. 30th Conference of the Canadian Medical and Biological Engineering Society, Toronto, Canada, 2007, M0100.

[15] R. N. Khushaba. A. Al-Ani, and A. Al-Jumaily, "Orthogonal fuzzy neighborhood discriminant analysis for multifunction myoelectric hand control", IEEE Transactions on Biomedical Engineering, vol. 57, no. 6, pp. 1410-1419, 2010.

[16] J. Ye, R. Janardan, Q. Li, and H. Park, "Feature reduction via generalized uncorrelated linear discriminant analysis", IEEE Transactions on Knowledge and Data Engineering, vol. 18, no. 10, pp. 1312-1322, 2006.

[17] H. Xiaofei, C. Deng, Y. Shuicheng, and H. J. Zhang, "Neighborhood preserving embedding", In Proc. 10th IEEE International Conference on Computer Vision, Beijing, China, 2005, pp. 1208-1213.

[18] X. He and P. Niyogi, "Locality preserving projections", In Proc. Advances in Neural Information Processing Systems 16 (NIPS), Vancouver, Canada, 2003.

[19] D. Cai, X. He, K. Zhou, J. Han, and H. Bao, "Locality sensitive discriminant analysis", In Proc. International Joint Conference on Artificial Intelligence, Hyderabad, India, 2007, pp. 708-713.

[20] X. He, S. Yan, Y. Hu, P. Niyogi, and H. J. Zhang, "Face recognition using laplacianfaces", IEEE Transactions on Pattern Analysis and Machine Intelligence, vol. 27, no. 3, pp. 328-340, 2005.

[21] C. C. Chang and C. J. Lin, "LIBSVM: A library for support vector machines", Available Online: http://www.csie.ntu.edu.tw/ cjlin/libsvm/, 2001.

[22] G. B. Hunag, Q. Y. Zhu, and C. K. Siew, "Extreme learning machine: theory and applications", Neurocomputing, vol. 70, pp. 489-501, 2006.

[23] M. Sugiyama, "Dimensionality reduction of multimodal labeled data by local Fisher discriminant analysis", Journal of Machine Learning Research, vol. 8, pp. 1027-1061, 2007.

[24] Z. P. Chen, J. H. Jiang, Y. Li, Y. Z. Liang, and R. Q. Yu, "Fuzzy linear discriminant analysis for chemical data sets", Chemometrics and Intelligent Laboratory Systems, vol. 45, no. 1-2, pp. 295-302, 1999. 